\title{
Global transcriptome analysis of Huperzia serrata and identification of critical genes involved in the biosynthesis of huperzine $A$
}

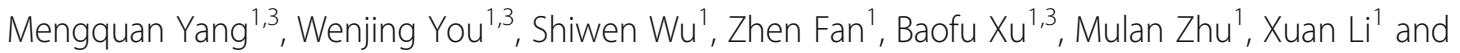
Youli Xiao ${ }^{1,2,3^{*}}$ (D)

\begin{abstract}
Background: Huperzia serrata $(H$. serrata) is an economically important traditional Chinese herb with the notably medicinal value. As a representative member of the Lycopodiaceae family, the $\mathrm{H}$. serrata produces various types of effectively bioactive lycopodium alkaloids, especially the huperzine A (HupA) which is a promising drug for Alzheimer's disease. Despite their medicinal importance, the public genomic and transcriptomic resources are very limited and the biosynthesis of HupA is largely unknown. Previous studies on comparison of 454-ESTs from H. serrata and Phlegmariurus carinatus predicted putative genes involved in lycopodium alkaloid biosynthesis, such as lysine decarboxylase like (LDC-like) protein and some CYP450s. However, these gene annotations were not carried out with further biochemical characterizations. To understand the biosynthesis of HupA and its regulation in $\mathrm{H}$. serrata, a global transcriptome analysis on $\mathrm{H}$. Serrata tissues was performed.
\end{abstract}

Results: In this study, we used the Illumina Highseq4000 platform to generate a substantial RNA sequencing dataset of H. serrata. A total of $40.1 \mathrm{~Gb}$ clean data was generated from four different tissues: root, stem, leaf, and sporangia and assembled into 181,141 unigenes. The total length, average length, N50 and GC content of unigenes were 219,520,611 bp, 1,211 bp, 2,488 bp and 42.51\%, respectively. Among them, 105,516 unigenes (58.25\%) were annotated by seven public databases (NR, NT, Swiss-Prot, KEGG, COG, Interpro, GO), and 54 GO terms and 3,391 transcription factors (TFs) were functionally classified, respectively. KEGG pathway analysis revealed that 72,230 unigenes were classified into 21 functional pathways. Three types of candidate enzymes, LDC, CAO and PKS, responsible for the biosynthesis of precursors of HupA were all identified in the transcripts. Four hundred and fifty-seven CYP450 genes in $\mathrm{H}$. serrata were also analyzed and compared with tissue-specific gene expression. Moreover, two key classes of CYP450 genes BBE and SLS, with 23 members in total, for modification of the lycopodium alkaloid scaffold in the late two stages of biosynthesis of HupA were further evaluated.

Conclusion: This study is the first report of global transcriptome analysis on all tissues of H. serrata, and critical genes involved in the biosynthesis of precursors and scaffold modifications of HupA were discovered and predicted. The transcriptome data from this work not only could provide an important resource for further investigating on metabolic pathways in H. serrata, but also shed light on synthetic biology study of HupA.

Keywords: Transcriptome, Huperzia serrata, Biosynthetic pathway, Huperzine A, Lycopodium alkaloid

\footnotetext{
* Correspondence: ylxiao@sibs.ac.cn

${ }^{1}$ CAS Key Laboratory of Synthetic Biology, CAS Center for Excellence in

Molecular Plant Sciences, Institute of Plant Physiology and Ecology, Shanghai

Institutes for Biological Sciences, Chinese Academy of Sciences, Shanghai

200032, China

${ }^{2}$ CAS-JIC Centre of Excellence in Plant and Microbial Sciences, Shanghai

200032, China

Full list of author information is available at the end of the article
}

(c) The Author(s). 2017 Open Access This article is distributed under the terms of the Creative Commons Attribution 4.0 International License (http://creativecommons.org/licenses/by/4.0/), which permits unrestricted use, distribution, and reproduction in any medium, provided you give appropriate credit to the original author(s) and the source, provide a link to the Creative Commons license, and indicate if changes were made. The Creative Commons Public Domain Dedication waiver (http://creativecommons.org/publicdomain/zero/1.0/) applies to the data made available in this article, unless otherwise stated. 


\section{Backgroud}

Huperzia serrata (H. serrate) is a model member of the Huperzia (Phlegmariurus) genus which belongs to the plant Family Lycopodiaceae, with a total of about 500 species worldwide $[1,2]$. The whole plant of $H$. serrata, named Qian Ceng Ta (in Chinese), is one of the oldest medicinally important traditional Chinese herbs since 739 (during the Tang Dynasty) and has been extensively used for the treatment of a number of ailments, including contusions, strains, swellings, schizophrenia, myasthenia gavis and organophosphate poisoning [3]. These pharmaceutical applications of $H$. serrata are mainly due to its biologically active lycopodium alkaloids. The four classes of lycopodium alkaloids with diverse chemical structures include lycopodine-type, lycodine-type, fawcettimine-type, and a set of miscellaneous-type compounds have been isolated from the $H$. serrata, which makes it a unique model plant for studying the biosynthesis of lycopodium alkaloids [4].

Among the lycopodium alkaloids, Huperzine A (HupA), one of the pyridine skeleton lycodine, was first isolated from $H$. serrata in 1986 [5] and was found to possess potent acetylcholine esterase inhibition (AChEI) [6, 7]. Since then, HupA had been clinically used for the treatment of Alzheimer's disease (AD) for years in China. Due to the medicinal importance of $H$. serrata and its economic value of bioactive ingredients, like HupA, this plant has been extensively harvested and is in danger of extinction in China. To overcome the issue, a lot of efforts have been put on plant cultivation and tissue culture for $H$. serrata production, but these approaches are rarely successful. Even the first transcriptomic data generated from 454-GS platform have been released for 6 years $[8,9]$, there are less than 90 nucleotide sequences are available in the National Center for Biotechnology Information (NCBI) databases (December, 2016). Using homologous cloning strategy, there were only three types of enzymes, lysine decarboxylase (LDC) [10, 11], copper amine oxidase (CAO) [12], and type III polyketide synthase (PKS) [13-15] were identified from $H$. serrata. Feeding experiments provided evidence that lycopodium alkaloids were secondary metabolites of lysine [16]. The first two biosynthetic steps for lycopodium alkaloids were generally believed involving the decarboxylation of lysine yields cadaverine catalyzed by LDC followed by the oxidative deamination of the latter to 5-aminopentanal catalyzed by $\mathrm{CAO}$ and then spontaneously to piperidine (Fig. 1) [17]. In the secondary stage of biosynthetic pathway, piperideine was reacted with 3-oxoglutaric acid which was generated from malonylCoA and followed by decarboxylation then formed pelletierine. Intramolecular tandem cyclization through phlegmarine would produce the key metabolite, lycodane, which contained the unique tetracyclic skeleton. From this basic intermediate, four classes of lycopodium alkaloids would be derived by further modification and oxidation

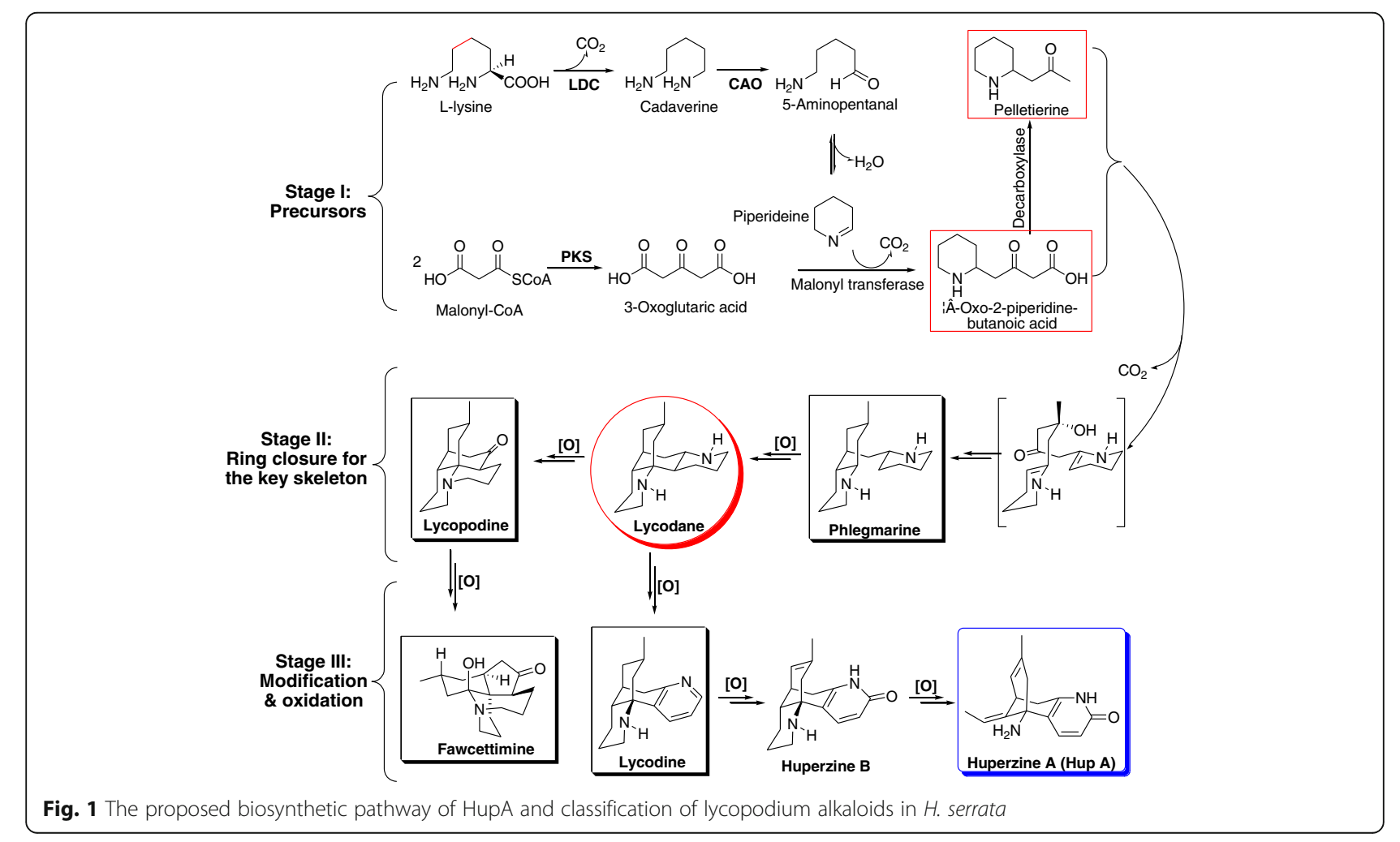


(Fig. 1). This stage of ring cyclization, rearrangement, modification and oxidation were most likely catalyzed by cytochrome P450s (CYP450s).

The limited transcriptomic data hamper the biosynthetic study of active lycopodium alkaloids in $H$. serrata and the biosynthetic pathway of HupA remains to be elucidated. The HiSeq4000 platform is the most efficient for highthroughput next-generation RNA-Seq which was used for transcriptomic profiling of non-model organisms with no available genomic data. In the current study, a global transcriptome analysis was designed to investigate the full gene contents of $H$. serrata and characterize their expression profiles in differentiated tissues (root, stem, leaf, and sporangia). Our work produced a total of 300 million sequence reads (40.1 $\mathrm{Gb}$ of clean data), resulting in 181,141 assembled unigenes. Genes involved in the biosynthesis of the HupA precursor and late stage were identified and predicted, the results of current work will serve as a valuable public resource facilitating the synthetic biology research on bioactive lycopodium alkaloids of traditional herbs.

\section{Results and discussion}

\section{Sample preparation, and Illumina sequencing}

To characterize the expressed sequences and discover the full gene contents in $H$. serrata, the study was designed to perform deep sequencing on RNA of all four differentiated tissues from it. Root, stem, leaf, and sporangia samples were firstly collected from the first $H$. serrata plant (Hs-1) followed by another two stem and leaf samples from the second one (Hs-2) as complements (Fig. 2). RNA-seq libraries were prepared from the collected tissues, and performed on Illumina HiSeq4000 system with a pair-end read length of 150 base pairs (bp). A total of 300 million raw reads (approximate 45.1 $\mathrm{Gb})$ were generated for all four $H$. serrata tissues, whereas those from roots, stems (2 samples), leaves (2 samples), and

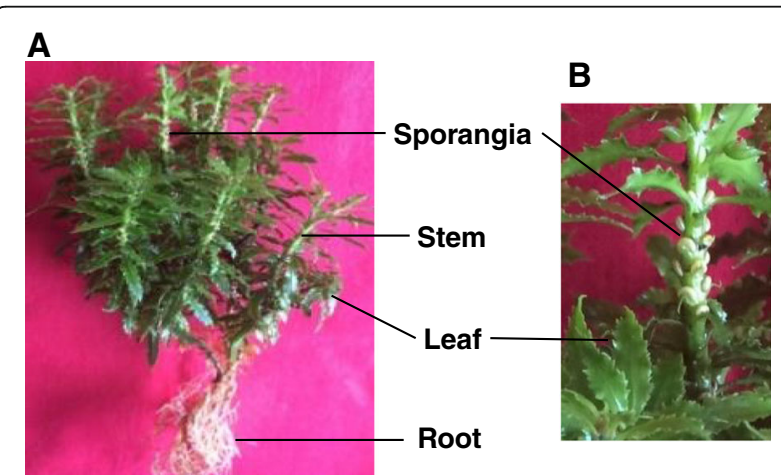

Fig. 2 H. serrata used in this study. a Image of whole-plant. b Enlarged image for tissues of sporangia (SP) and leaf (LF). The name of each tissue was labeled with bold words sporangias were $7.59 \mathrm{~Gb}, 7.59 \mathrm{~Gb}$ (7.35 Gb from Hs-2), $7.59 \mathrm{~Gb}$ (7.35 Gb from $\mathrm{Hs}-2)$, and $7.59 \mathrm{~Gb}$, respectively (Table 1).

\section{De novo transcriptome assembly, gene expression comparison among tissues, and comparison with previous 454-ESTs report}

After reads filtering to remove adaptor sequences and low-quality reads, high-quality clean reads from the four tissues were pooled together for de novo assembly using Trinity [18], and a total of 830,623 contigs were generated with a mean size of $812 \mathrm{bp}$. Hence, 181,141 unigenes were obtained with an average size of 1,211 bp ranging from $301 \mathrm{bp}$ to $3,000 \mathrm{bp}$ in size. In total, 105,516 unigenes $(58.25 \%)$ were longer than $500 \mathrm{bp}$, and 70,746 unigenes (39.06\%) were longer than $1.0 \mathrm{~kb}$ (Fig. 3).

In order to get the gene expression profiles of each individual tissue, all the data from different tissues was firstly analyzed by cluster dendrogram (Additional file 1). Based on the relationship between the six data sets, four tissue data sets from the same plant (Hs-1) were picked for following tissue-specific analysis and further mapped back to the assembled unigenes using bowtie 2 with their clean reads [19]. While the sporangia tissue has the highest numbers of gene expressed, all the four tissues shared a similar distribution in gene expression level (Fig. 4). There were 130,297 unigenes (71.9\%) expressed above the threshold level (fragment per kilobase per million mapped reads $(F P K M) \geq 0.5)$ in the four tissues, of which 56,958 genes were common expressed in all the four tissues (Fig. 4c). Generally, the sporangia expressed the largest number of tissue-specific unigenes $(18,317)$, followed by the stem $(8,222)$, root $(7,242)$, and leaf $(5,506)$.

A total of $267,314,012$ high quality reads is three orders of magnitude greater than the previous reported from $H$. serrata, in which 140,930 high quality reads generated from $57 \mathrm{Mb}$ data using Roche 454 GS FLX Titanium

Table 1 Overview of the sequencing and assembly of transcriptome of $H$. serrata

\begin{tabular}{lll}
\hline Items & No. of reads (bytes) & No. of bases (bp) \\
\hline Leaf-1 (from Hs-1) & $50,627,568$ & $7,594,135,200$ \\
Stem-1 (from Hs-1) & $50,627,508$ & $7,594,126,200$ \\
Root-1 (from Hs-1) & $50,627,472$ & $7,594,120,800$ \\
Sporangia-1 (from Hs-1) & $50,627,354$ & $7,594,103,100$ \\
Leaf-2 (from Hs-2) & $48,994,336$ & $7,349,150,400$ \\
Stem-2 (from Hs-2) & $48,994,084$ & $7,349,112,600$ \\
Total raw data & $300,498,322$ & $45,074,748,300$ \\
Total high-quality data & $267,314,012$ & $40,097,101,800$ \\
Average length of unigenes & 1,211 bp & $219,520,611$ \\
Unigenes $\geq 300$ bp & 181,141 & \\
N50 (bp) & 2,488 & \\
\hline
\end{tabular}




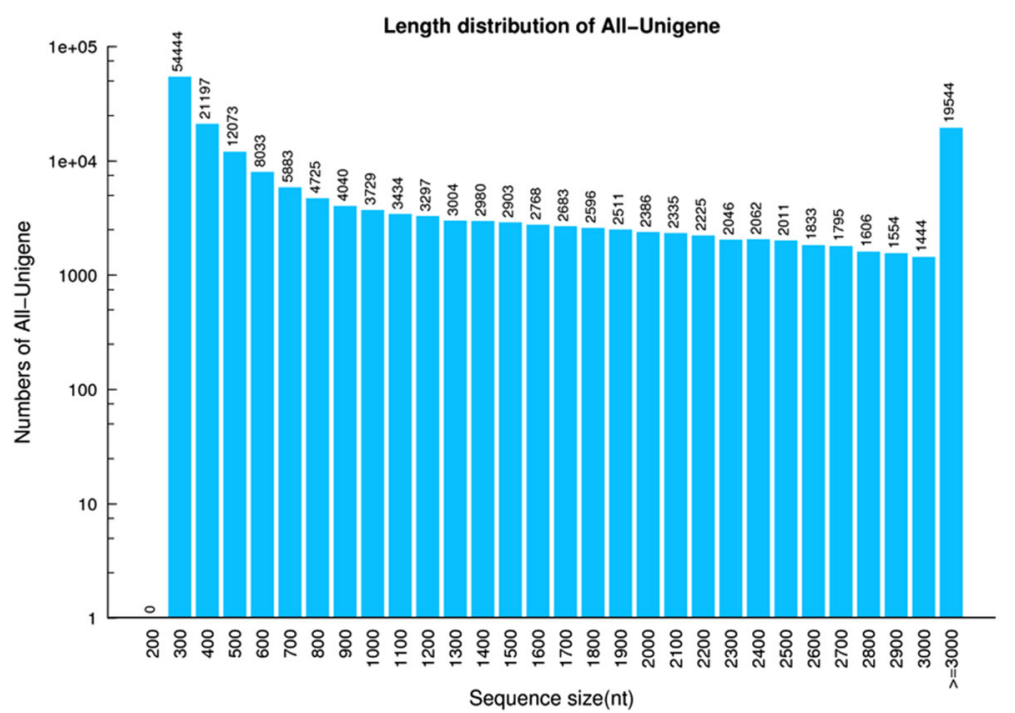

Fig. 3 Length distribution of assembled unigenes. Out of the 181,141 unigenes, 105,516 unigenes (58.25\%) were longer than 500 bp, and 70746 unigenes (39.06\%) were longer than $1.0 \mathrm{~kb}$

system (Additional file 2) [8, 9]. The reads generated from 454 platform were assembled to 14,085 contigs using GS De Novo Assembler software v2.0.01 (454 Life Sciences, Roche) with an average length of $608 \mathrm{bp}$. With the higher throughput of Illumina Hiseq platform, more and longer contigs were produced. Our clean reads were assembled to 830,623 contigs, with the average length is $812 \mathrm{bp}$. 181,141 unigenes were generated and $58.25 \%$ of them were annotated at last, to some extent, showing the disadvantage of short-read sequence assembly was avoided by more sequence data.

\section{Function annotation of transcriptome for $\mathrm{H}$. serrata}

All assembled unigenes were annotated with 7 functional databases (NCBI non-redundant nucleotide database $(\mathrm{Nr})$,
NCBI non-redundant protein database (Nt), Gene Ontology databases (GO), Clusters of Orthologous Groups of proteins database (COG), Kyoto Encyclopedia of Genes and Genomes (KEGG), Swiss Institute of Bioinformatics (Swiss-Prot), and InterPro) (Table 2). In total, 58.25\% $(105,516)$ annotated sequences were identified, which was higher than the 454-EST level reported $(44.3 \%)[8,9]$. These assembled transcripts were mapped to the 7 public databases, and the amount of transcripts which were aligned to each database were shown in Fig. 5a. Among the annotated sequences, the species with the highest number of best hits were Selaginella moellendorffii (22.78\% matched genes) and Physcomitrella patens (16.05\% matched genes) (Fig. 5b). These results are consistent since $S$. moellendorffii and $P$. patens are the species closest to H.serrata with sequenced
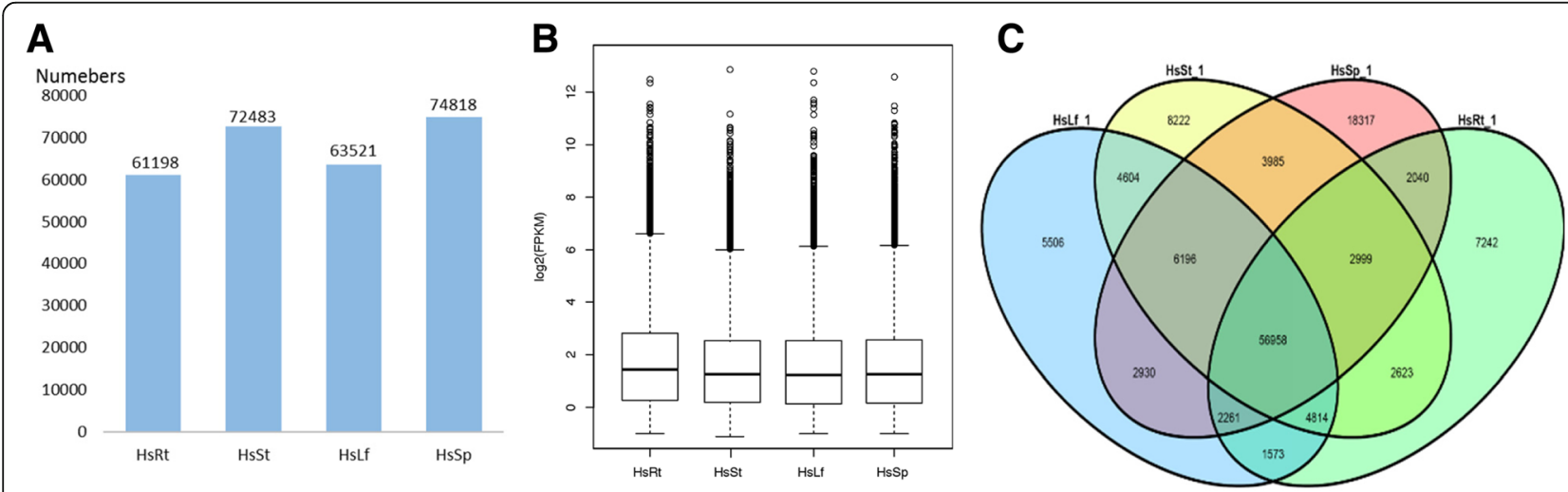

Fig. 4 Unigenes expressed number and level on different tissues. a The number of expressed genes (FPKM $\geq 0.5$ ) in each tissue was labeled over the bars. $\mathbf{b}$ Expressional levels of unigenes of all the tissues. $\mathbf{c}$ Venn diagram of expressed unigenes in four tissues. A total of 130,297 unigenes (71.9\%) were expressed, of which 56,958 genes were common expressed in all four tissues. Unigenes with FPKM $\geq 0.5$ were considered as expressed in each tissue 
Table 2 Summary of unigenes annotations

\begin{tabular}{lllc}
\hline Database & Total unigenes & Annotated unigenes & Percentage (\%) \\
\hline $\mathrm{Nr}$ & 181,141 & 94,338 & 52.08 \\
$\mathrm{Nt}$ & 181,141 & 52,626 & 29.05 \\
Swiss-Prot & 181,141 & 64,634 & 35.68 \\
KEGG & 181,141 & 72,230 & 39.88 \\
COG & 181,141 & 46,372 & 25.60 \\
Interpro & 181,141 & 77,671 & 42.88 \\
GO & 181,141 & 45,780 & 25.27 \\
Overall & 181,141 & 105,516 & 58.25 \\
\hline
\end{tabular}

genomes, in which both of them are members of ancient vascular plant lineage, and $P$. patens is a lycophyte, the oldest extant division of the vascular plants.

\section{GO and KEGG classification}

The function annotation was conducted on the basis of homology. GO assignments were used to predict the functions of H.serrata unigenes by classifying them into three major categories: biological process, cellular component and molecular function (Fig. 6a). Based on sequence homology, the 45,780 unigenes annotated in the GO database were categorized into 54 functional groups. Among these groups, "cellular process and metabolic process", "cell and cell part", and "binding and catalytic activity" terms were dominant within each of these categories, respectively. Additionally, we noticed that many genes were classified into the "single-organism process", "organelle" and "membrane" groups, whereas only a few genes were classified into the "extracellular matrix part", "virion" and "metallochaperone activity" groups. The high percentage of genes from the "metabolic process" $(24,605)$ and "catalytic activity" $(23,841)$ might serve as good candidates for identification of novel genes that participated in the lycopodium biosynthesis pathway. KEGG is a classification method based on pathways for systematic analysis of gene function in terms of the networks of gene products. To identify biochemical pathways, 72,230 genes were mapped to 136 KEGG pathways which were classified into six main categories (Fig. 6b). Most genes were annotated with metabolic function $(60.47 \%, 43,674)$, followed by "genetic information processing" (29.66\%, 21,427). The pathways with most representation were "global and overview maps" (16,563 unigenes, 22.93\%), "translation" (9,249 unigenes, $12.80 \%)$, and "carbohydarte metabolism" $(6,342$ unigenes, $8.78 \%)$.

These annotation and classifications provided a resource for investigating specific pathways in $H$. serrata, such as lycopodium alkaloids biosynthetic pathway. Lycopodium alkaloids are lysine derivated alkaloids, therefore, the $3,768,1,858$, and 1,333 unigenes clustered into "amino acid metabolism", "biosynthesis of other secondary metabolites", and "metabolism of terpenoids and polyketides" might potentially be involved in the biosynthesis and metabolism of lycopodium alkaloids.

\section{Transcription factor (TF)}

With functional annotation results, we detected 94,510 CDS (coding DNA sequence) firstly, and continually found 18,139 CDS predicted by ESTScan with the remaining unigenes. We also detected 1,478 SSR which distributed on 26,579 unigenes, and predicted 2,975 TF coding unigenes with family classification (Fig. 7 and Additional file 3). Among the TF types, MYB (404 members), MYB-related (280 members), bHLH (238 members) and $\mathrm{C} 3 \mathrm{H}$ (215 members) proteins were the most abundant above 200 members, with the largest (MYB) having 404 members in comparison with 155 and 197 MYB genes in rice and Arabidopsis, respectively [20]. Functions of MYB proteins are involved in secondary metabolism, regulation of gene expression and response to environmental stresses [21].
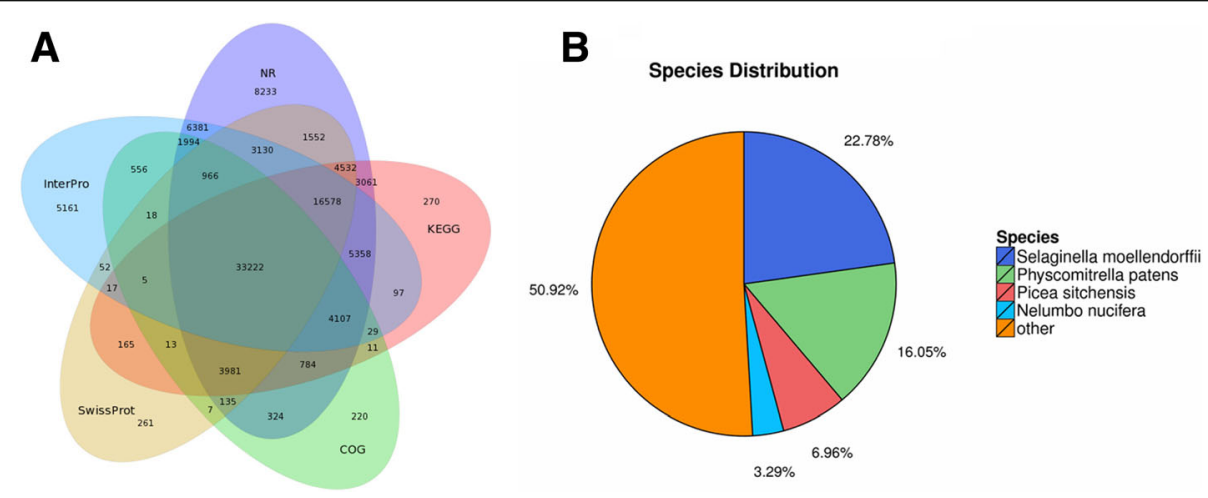

Fig. 5 Results summary for the sequence homology search against the NCBI NR database. a Venn diagram of transcripts detected in NR, COG, KEGG, Swissprot and Interpro. b Distribution of annotated species 

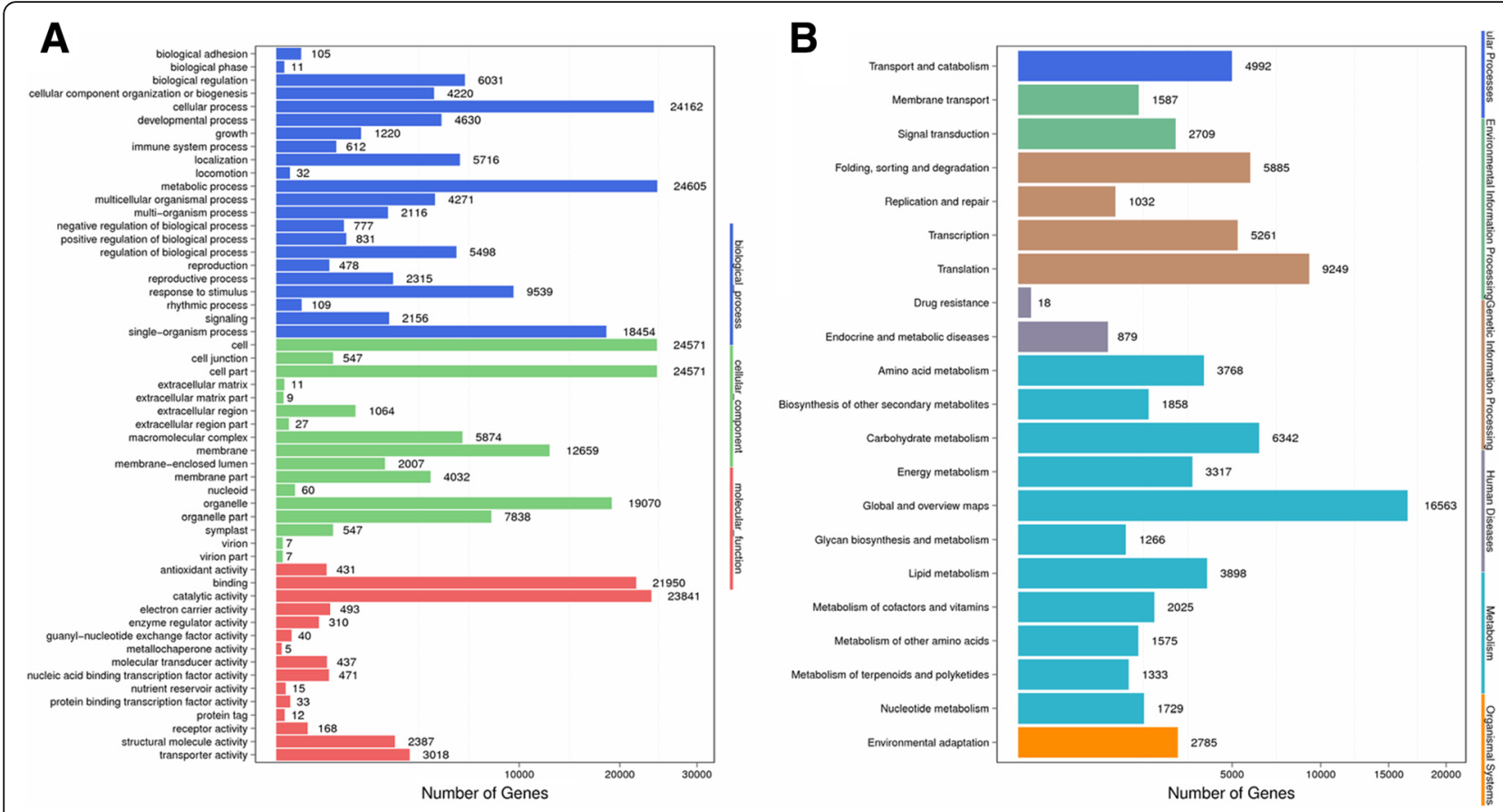

Fig. $6 \mathrm{GO}$ and KEGG analysis. a Histogram of GO classifications. The results are summarized in the following three main categories: (1) biological process, (2) cellular component and (3) molecular function. b Pathway assignment based on KEGG. The bottom $x$-axis indicates the number of genes in a specific category. The left $y$-axis indicates the clustered function groups, the right $y$-axis indicates the specific category of genes in the main category

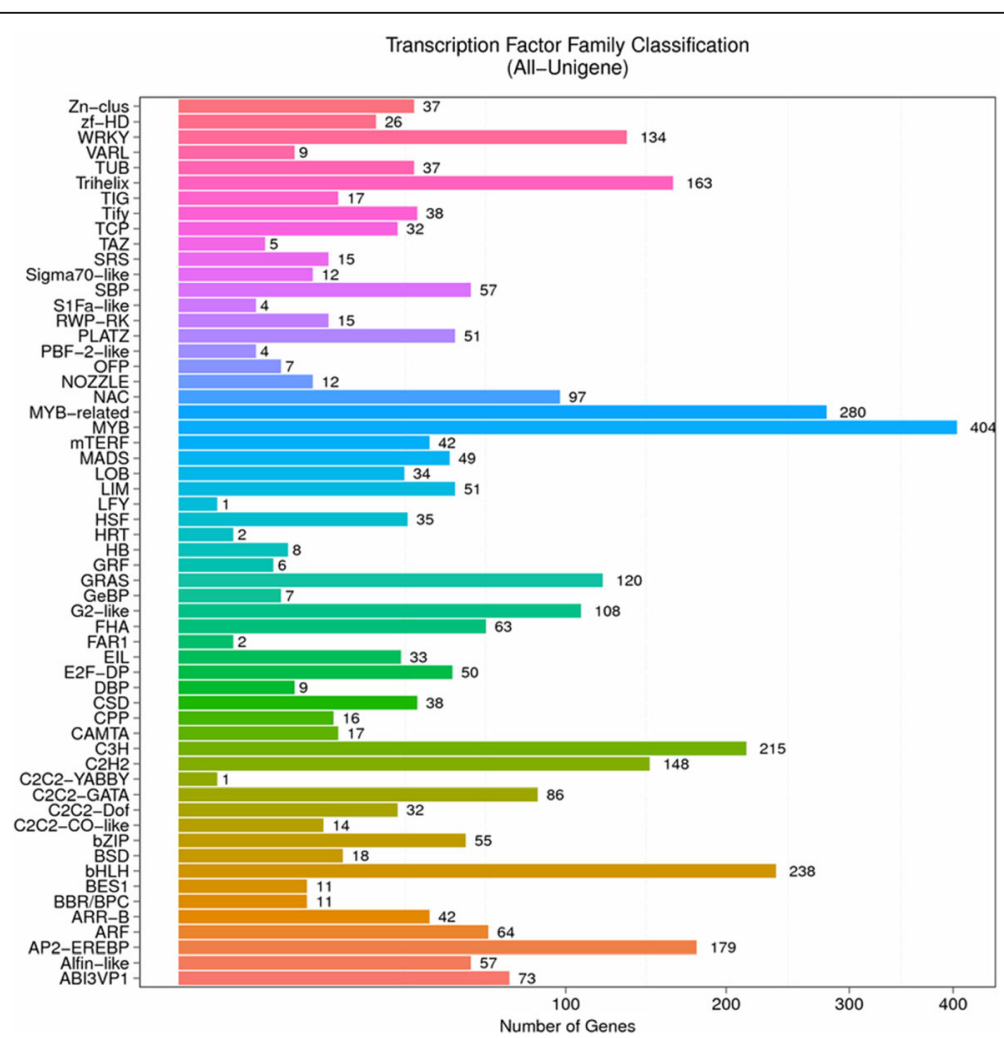

Fig. 7 Transcription Factor (TF) family classification of unigenes. Number of unigenes related to TFs in each families, MYB, MYB-related, bHLH, and $\mathrm{C} 3 \mathrm{H}$ proteins were the most abunant 


\section{Genes involved in the biosynthesis of lycopodium alkaloids and HupA}

To identify active biological pathways in $H$. serrata, the sequences were mapped to the reference pathways in the KEGG. Most metabolic related genes were involved in primary metabolism, such as carbohydrate metabolism, lipid metabolism, amino acid metabolism, and energy metabolism. The 1,858 genes were grouped in the "Biosynthesis of other secondary metabolites" category, which were important contributors to highly valuable secondary metabolite biosynthesis. Especially 140 genes were mapped to the "tropane, piperidine and pyridine alkaloid biosynthesis". The 119 genes were mapped to the "isoquinoline alkaloid biosynthesis" would be useful for defining metabolic pathways and metabolic genes for lycopodium alkaloids synthesis in H. serrata (Fig. 6b).

As shown in the Fig. 1, the biosynthesis pathway of lycopodium alkaloids as well as HupA, was proposed within three stages, formation of the precursors, ring closure for the tetracyclic basic skeleton, and modification and oxidation. In the first stage of producing necessary precursors starting from lysine and malonyl-CoA as the initiated substrates, it was believed that LDC, CAO, and PKS were involved in the bioconversion. Using degenerate primers strategy based on the sequence homology, these three types of genes/enzymes from $H$. serrata have been identified by us and other research groups followed by biochemically characterization [10-15]. All these genes were also found in our transcriptomic data (Table 3 and Additional file 4 with the detailed sequences). These gene's homologues and their multiple-tissues specific expression patterns were also analyzed by phylogenetic tree and heat map (Additional file 5). Besides, we performed phylogenetic analysis for LDC, CAO, PKS with their reported homologues and accession numbers of selected genes (Fig. 8a-c and Additional file 6). It's clear that the eukaryotic LDCs were monophyletic origin from the phylogenetic analysis which was consistent to previous study (Fig. 8a) [10]. As Lycophyte, Angiosperm, and Legume were responsible for biosynthesis of lycopodium, nuphar, and quinolizidine alkaloids, respectively, the LDC clade formed from these three distinct plant lineages were distant from each other which indicated a convergent evolution of the Lys-derived alkaloid production. In conclusion, these genes were located in the

Table 3 Possible unigenes and encoding enzymes involved in biosynthesis of HupA precursor

\begin{tabular}{|c|c|c|}
\hline ene & Degenerate primes method & Unigenes from transcriptomic data \\
\hline$\overline{L D C}$ & $\begin{array}{l}\text { AB915696.1, AB915697.1 } 1^{[10]} \\
\text { and HsLDC-X1 to }-X 6^{[11]}\end{array}$ & unigene96617 and unigene94988 \\
\hline CAO & $\mathrm{HsCAO}{ }^{[12]}$ & CL4248.1, CL4248.2, CL4248.3 \\
\hline KS & $\begin{array}{l}\text { HsPKS1 (ABI94386.1 })^{[13]} \\
\text { HsPKS2 }^{[14]} \text {, and HsPKS3 } \\
\end{array}$ & $\begin{array}{l}\text { unigene393, CL2724.2, and } \\
\text { unigene394 }\end{array}$ \\
\hline
\end{tabular}

lower creatures. Generally, these three types of biochemically confirmed genes were either exact same or had high identity to the corresponding transcripts from the phylogenetic tree analysis. The newly discovered homologue genes provide more enzymatic parts in the toolbox for synthetic biology usage in the biosynthesis of precursors of HupA.

In the late two stages of biosynthesis of lycopodium alkaloids and HupA, most of steps were proposed through oxidative modifications which indicated that CYP450s were candidates. To identify novel genes encoding enzymes possibly associated with the catalytic modification, 457 putative CYP450 sequences with 199 full-length ones were obtained which was about 5 fold greater than the 96 CYP450 members in 454-ESTs data [9]. These P450 sequences were annotated and classified into different CYP families by using phylogenetic analysis (Fig. 9a and Additional file 7). The middle stage of conduction of the key scaffold, like phlegmarine and lycodane, through intramolecular tandem cyclization with new $\mathrm{C}-\mathrm{C}$ bond formation, shares a similar formation of the methylenedioxy bridge in berberine synthesis with the gene encoded by AAU20771.1 from Thalictrum tuberosum [22]. With this gene as the probe, a phylogenetic tree was generated and 11 homologues were identified as the berberine bridge enzyme (BBE) (Additional file 7 and Additional file 8). The heat map was also analyzed with differential tissues (Fig. 9b). In addition, It was also noteworthy that 12 CYP450s belong to a group with CYP450 (Q05047.1) encoding the enzyme secologanin synthase (SLS) based on the phylogenetic analysis (Additional file 7 and Additional file 9), which catatlyzed the oxidative cleavage of loganin into secologanin [23]. From the tissue-specific analysis (Fig. 9c), these candidate CYP450s were expressed more abundantly in leaves than other organs, which was consistent with the organ-specific accumulation pattern of lycopodium alkaloids [24]. These two classes of CYP450s may play key roles in biosynthesis of lycopodium alkaloid as well as HupA and will be considered as lead candidates in the late two stages of ring formation and oxidation.

\section{Validation of RNA-Seq results by qRT-PCR}

qRT-PCR was performed to validate four types of differentially expressed genes identified by RNA-Seq in the four tissues of $H$. serrata. With tubulin (Unigene50132) as the internal control, 7 selected genes involved in HupA biosynthesis, including HsLDC, HsCAO, HsPKS and CYP450s (SLS and BBE classes), were evaluated. The validation results were consistent with the gene expression patterns identified by RNA-Seq (Additional file 10). The expression levels of Unigene25121 were slightly higher based on qRT-PCR than RNA-Seq. These results highlighted the fidelity and reproducibility of the RNA-Seq analysis used in the present study. 


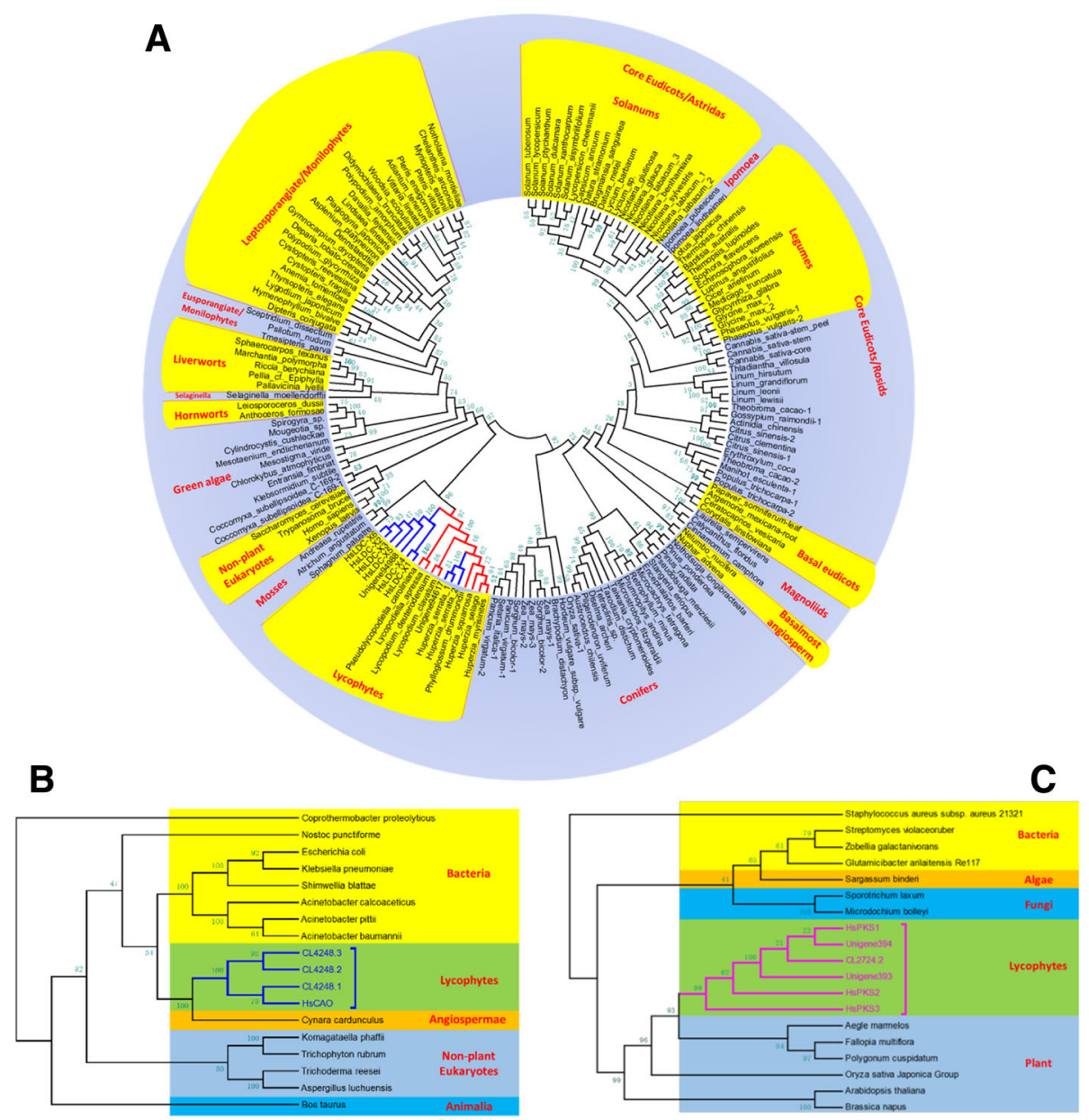

Fig. 8 Phylogenetic analysis of LDC, CAO, PKS. Unrooted neighbor-joining phylogenetic tree of selected LDC, CAO, PKS proteins. Bootstrap values (1000 replicates) were shown above each branch. a Phylogenetic tree of LDC amino acid sequences from eukaryotes. Phylogenetic tree of CAO (b) and PKS (c) amino acid sequences. The blue branch lines represented genes in this study and biochemically confirmed [11-15]

\section{Conclusion}

We conducted deep RNA-sequencing analysis on four tissues of $H$. serrata and a total of 300 million reads were generated. 181,141 unigenes were assembled, in which nearly $60 \%$ were successfully annotated. The data offered a comprehensive coverage of $H$. serrata transcriptome and paved the way for elucidation of the biosynthesis pathway of lycopodium alkaloids, like HupA. The three types of biochemically confirmed enzymes in the biosynthesis of the precursors, LDC, CAO and PKS, were all identified in this study. Moreover, a large number of CYP450s involved in the secondary metabolic pathway were evaluated. We predicted that the BBE and SLS types of CYP450s were involved the ring closed and cleavage in the biosynthesis of HupA. Further studies are needed to elucidate the CYP450s involved in the ring formation and oxidative modification of the biosynthesis of HupA. The study provides valuable resources for bioengineering and synthetic biology study of the lycopodium alkaloids.

\section{Methods}

Plant materials and treatments

Two independent $H$. serrata plants (Hs-1 and Hs-2) were collected from Xiangxi, Hunan, China, in December 2015 and identified by Dr. Zhu Mulan. The plants were carefully rinsed in running tap water and soil was removed by hand. Root, stem, leaf, and sporangia, were kept in collection tubes immediately after separated from the plant and immersed in liquid nitrogen, and then stored at $-80{ }^{\circ} \mathrm{C}$ until further use.

\section{RNA isolation, cDNA library construction and Illumina sequencing}

Total RNA was extracted from four different tissues of $H$. serrata, including root, stem, leaf and sporangia with TIANGEN RNAprep Pure Plant Kit. DNase I was used to digest contaminated DNA. The purified total RNA was quantified using Nanodrop, Agilent 2100, and agrose gel 

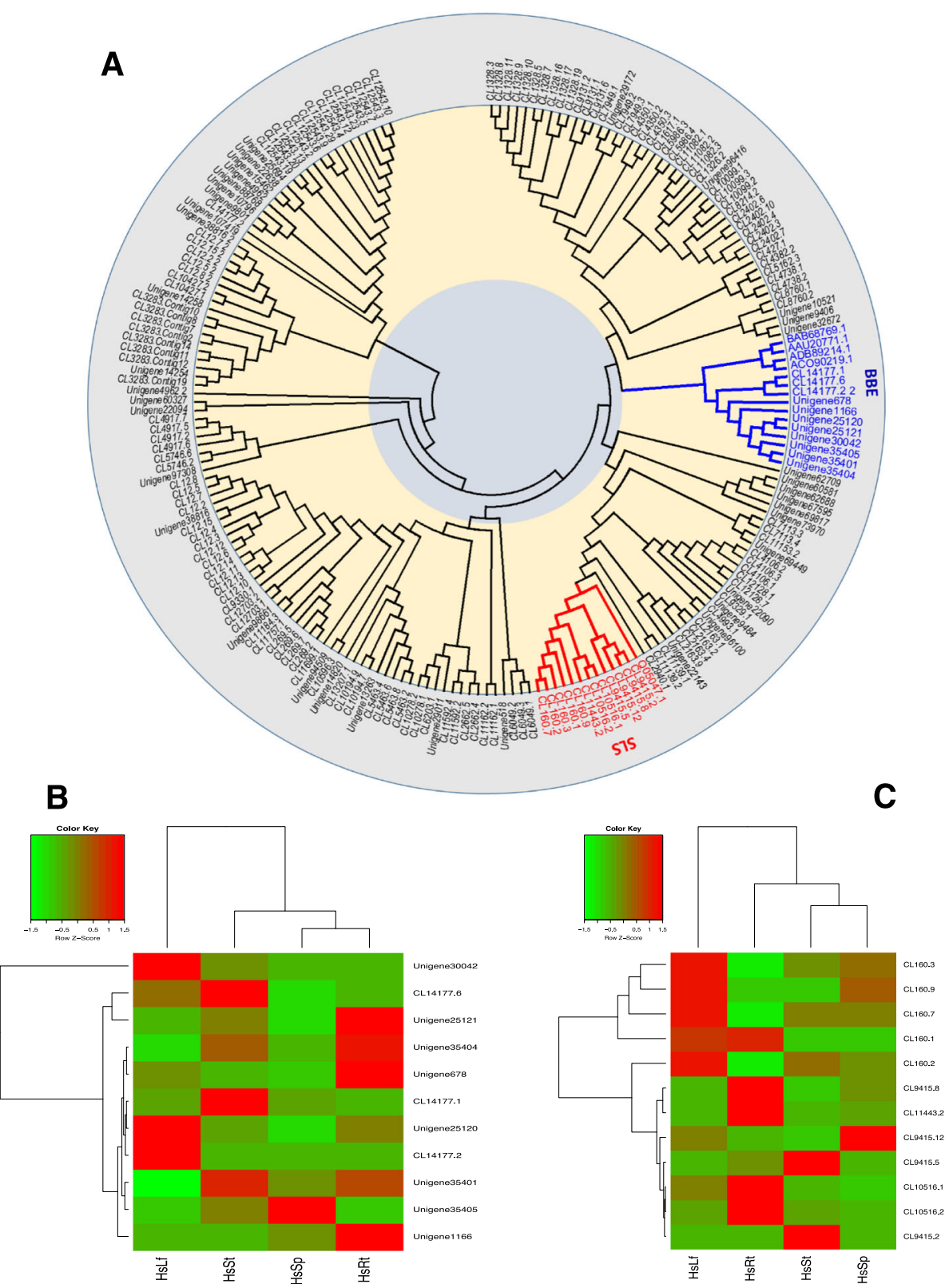

Fig. 9 Phylogenetic analysis of CYP450s and the heat map of BBE and SLS. a Phylogenetic analysis of 199 full-length CYP450s. The tissue-specific heat map of BBE (b) and SLS (c). The detail information about all the CYP450s including BBE and SLS were listed in Additional file 7-9

electrophoresis. Oligo(dT) was used to isolate mRNA followed by fragmentation. cDNA was synthesized using the mRNA fragments as templates. Short fragments were purified and resolved with EB buffer for end reparation and single nucleotide A (adenine) addition followed by connected with adapters. The suitable fragments were selected for the PCR amplification. Agilent 2100 Bioanaylzer and ABI StepOnePlus Real-Time PCR System were used in quantification and qualification of the sample library. The cDNA library was sequenced from both of 5' and 3' ends on the Illumina HiSeq4000 platform with paired-end sequecing length of $150 \mathrm{bp}$ according to the manufacturer's instructions.

De novo assembly and mapping of sequencing reads and analysis

Trinity was used to perform de novo assembly with clean reads that PCR duplication removed (in order to improve the efficiency), and Tgicl was used to cluster transcripts to unigenes. After assembly, clean reads were mapped to unigenes using Bowtie2 [19], and then gene expression level was calculated with RSEM [25]. To assess the gene 
expression abundance, the differentially expressional levels of unigenes in the four tissues were measured by FPKM values, with $\mathrm{FPKM} \geq 0.5$ used as a cut-off.

\section{Functional annotation and classification}

All assembled unigenes were searched against the $\mathrm{Nr}$ database and the SWISS-PROT database using BLASTX. Unigenes were also compared with the COG and KEGG using BLASTX. InterPro domains were annotated by InterProScan5 [23] and functional assignments were mapped onto GO database.

\section{Phylogenetic analysis}

Amino acid sequences were aligned using the CLUSTAL $\mathrm{W}$ program and evolution distances were computed using the Poisson correction method, and a NeighorJoining (NJ) tree was constructed with MEGA6. Bootstrap values which have been converted into the percentage obtained after 1000 replications are given on the branches

\section{Quantitative real-time PCR}

Quantitative real-time PCR (qRT-PCR) amplification was performed to validate the RNA-seq data with the designed primers (Additional file 10). The experiment was conducted with CFX Real-Time PCR Detection System (BIO-RAD, USA) using SYBR ${ }^{\circ}$ Premix Ex Taq ${ }^{\text {Tx }}$ kit (TaKaRa), and it was repeated three times. The mean value of three replicates was normalized using Tubulin (unigen50132) as the internal control. PCR mixtures (final volume, 25.0 $\mu \mathrm{L}$ ) contained $200 \mathrm{ng}$ of cDNA, $0.200 \mu \mathrm{M}$ each primer, $8.00 \mu \mathrm{L}$ of sterile water, and $12.5 \mu \mathrm{L}$ of SYBR Green Premix Ex Taq (TakaRa). The conditions for amplification were described as follows: 10 min denaturation at $95{ }^{\circ} \mathrm{C}, 40$ cycles of $95{ }^{\circ} \mathrm{C}$ for $10 \mathrm{~s}$, $57{ }^{\circ} \mathrm{C}$ for $20 \mathrm{~s}$, and $72{ }^{\circ} \mathrm{C}$ for $20 \mathrm{~s}$. Melting curves were determined ranging from $60^{\circ} \mathrm{C}$ to $95^{\circ} \mathrm{C}$ at $0.5^{\circ} \mathrm{C} / \mathrm{min}$.

\section{Additional files}

Additional file 1: All the multiple-tissues specific data from $\mathrm{H}$. serrata analyzed by cluster dendrogram. (PDF $81 \mathrm{~kb}$ )

Additional file 2: Summary of $H$. serrata RNA seq by two Next-Generation Sequencing Systems (PDF $44 \mathrm{~kb}$ )

Additional file 3: List of transcription factors (TFs) obtained from $\mathrm{H}$. serrata transcriptome. (XLS $194 \mathrm{~kb}$ )

Additional file 4: The ORF or amino acid sequences of three types (LDC, CAO, PKS) of enzymes identified from $\mathrm{H}$. serrata and found in the transcriptome. LDC (HsLDC-X1, HsLDC-X2, HsLDC-X3, HsLDC-X4, HsLDCX5, HsLDC-X6, AB915697.1, AB915696.1, Unigene94617, Unigene94988); CAO (HsCAO, CL4248.1, CL4248.2, CL4248.3); PKS (HsPKS1 (ABI94386.1), HsPKS2, HsPKS3, Unigene393, Unigene394, CL2724.2). (PDF 63 kb)

Additional file 5: LDC's, CAO's, and PKS's multiple-tissues specific expression patterns analysis by heat map. (PDF $63 \mathrm{~kb}$ )

Additional file 6: Selected genes for LDC's, CAO's, and PKS's phylogenetic analysis. (PDF $66 \mathrm{~kb}$ )
Additional file 7: Unrooted neighbor-joining phylogenetic tree of selected CYP450s (Bootstrap values (1000 replicates)). (PDF $45 \mathrm{~kb}$ )

Additional file 8: The amino acid sequences of 11 homologues identified as BBE. (PDF 33 kb)

Additional file 9: The amino acid sequences of 12 homologues identified as SLS. (PDF 35 kb)

Additional file 10: qRT-PCR expression analyses of $\mathrm{HsLDC}, \mathrm{HsCAO}$, HsPKS, and CYP450s. (PDF $36 \mathrm{~kb}$ )

\section{Abbreviations}

CAO: Copper amine oxidase; CDS: Coding DNA sequence; COG: Clusters of orthologous groups of proteins database; CYP450s: Cytochrome P450s; FPKM: Fragment per kilobase per million mapped reads; GO: Gene ontology databases; KEGG: Kyoto encyclopedia of genes and genomes; LDC: Lysine decarboxylase; NCBI: National center for biotechnology information; Nr: NCBI non-redundant nucleotide database; Nt: NCBI non-redundant protein database; PKS: Polyketide synthase; SwissProt: Swiss institute of bioinformatics

\section{Acknowledgements}

We thank Dr. Lei Lei for RNA sample preparation assistance.

\section{Funding}

This work was financially supported by the Science and Technology Commission of Shanghai Municipality (Grant 15JC1400402), CAS-JIC Centre of Excellence in Plant and Microbial Sciences (CEPAMS) funding, the "Thousand Talents Program" young investigator award, and the National Natural Science Foundation of China (Grant 21572243).

\section{Availability of data and materials}

The raw sequence data reported in this paper have been deposited in Genome Sequence Archive in BIG Data Center (http://bigd.big.ac.cn/gsa), Beijing Institute of Genomics (BIG), Chinese Academy of Sciences, under accession number: PRJCA000351 that are publicly accessible at http://gsa.big.ac.cn:80/preview/preview.action?code=0KDhCN95.

\section{Competing interests}

The authors declare that they have no competing interests.

\section{Authors' contributions}

YX conceived study and wrote manuscript. MY performed the most of experiments and data analysis. WY and SW performed qRT-PCR. ZF, BX and $\mathrm{XL}$ participated in data analysis and experiments coordination. $\mathrm{MZ}$ collected the samples. All authors read, commented, and approved the final manuscript.

\section{Consent for publication}

Not applicable

\section{Ethics approval}

Huperzia serrata is a wild plant source, and it could not be artificially cultivated in field. Huperzia serrata has not been listed in the appendices I, II and III of the Convention on the Trade in Endangered Species of Wild Fauna and Flora which was validated from Jan 2th, 2017 (website: https:/cites.org/eng/app/appendices.php). The collection of this plant materials comply seriously with the Chinese and international guidelines. Huperzia serrata in this case were collected from Xiangxi, Hunan, China. Researchers had deposited this member of family, Huperzia serrata, in the publicly Chinese herbarium Chinese Virture Herbarium (CVH) in 2008 with Herbarium: HUST BarcodelD:00019690 (website: http://www.cvh.ac.cn/en/spm/HUST/ 00019690).

\section{Publisher's Note}

Springer Nature remains neutral with regard to jurisdictional claims in published maps and institutional affiliations.

\section{Author details}

${ }^{1}$ CAS Key Laboratory of Synthetic Biology, CAS Center for Excellence in Molecular Plant Sciences, Institute of Plant Physiology and Ecology, Shanghai Institutes for Biological Sciences, Chinese Academy of Sciences, Shanghai 
200032, China. ${ }^{2}$ CAS-JIC Centre of Excellence in Plant and Microbial Sciences, Shanghai 200032, China. ${ }^{3}$ University of Chinese Academy of Sciences, Beijing 100039, China.

Received: 3 January 2017 Accepted: 10 March 2017

Published online: 22 March 2017

\section{References}

1. Christenhusz $M$, Zhang $X$, Schneider $H$. A linear sequence of extant families and genera of lycophytes and ferns. Phytotaxa. 2011;19(1):7-54.

2. Kitajima M, Takayama H. Lycopodium alkaloids: isolation and asymmetric synthesis. In: Alkaloid Synthesis. Berlin: Springer; 2011;1-31.

3. Ma X, Tan C, Zhu D, Gang DR, Xiao P. Huperzine a from huperzia species-an ethnopharmacolgical review. J Ethnopharmacol. 2007;113(1):15-34.

4. Ferreira A, Rodrigues M, Fortuna A, Falcão A, Alves G. Huperzine A from Huperzia serrata: a review of its sources, chemistry, pharmacology and toxicology. Phytochem Rev. 2016;15(1):51-85.

5. Liu J, Zhu Y, Yu C, Zhou Y, Han Y, Wu F, Qi B. The structures of huperzine $A$ and $B$, two new alkaloids exhibiting marked anticholinesterase activity. Can J Chem. 1986;64(4):837-9.

6. Tang $X$, Han $Y$, Chen $X$, Zhu $X$. Effects of huperzine $A$ on learning and the retrieval process of discrimination performance in rats. Acta Pharmacol Sin. 1986;7(6):507.

7. Tang X, De Sarno P, Sugaya K, Giacobini E. Effect of huperzine A, a new cholinesterase inhibitor, on the central cholinergic system of the rat. J Neurosci Res. 1989;24(2):276-85.

8. Luo H, Sun C, Li Y, Wu Q, Song J, Wang D, Jia X, Li R, Chen S. Analysis of expressed sequence tags from the Huperzia serrata leaf for gene discovery in the areas of secondary metabolite biosynthesis and development regulation. Physiol Plantarum. 2010;139(1):1-2.

9. Luo H, Li Y, Sun C, Wu Q, Song J, Sun Y, Steinmetz A, Chen S. Comparison of 454-ESTs from Huperzia serrata and Phlegmariurus carinatus reveals putative genes involved in lycopodium alkaloid biosynthesis and developmental regulation. BMC Plant Biol. 2010;10(1):1.

10. Bunsupa S, Hanada K, Maruyama A, Aoyagi K, Komatsu K, Ueno H, Yamashita M, Sasaki R, Oikawa A, Saito K, Yamazaki M. Molecular evolution and functional characterization of a bifunctional decarboxylase involved in lycopodium alkaloid biosynthesis. Plant Physiol. 2016;171(4):2432-44.

11. Xu B, Lei L, Zhu X, Zhou Y, Xiao Y: Identification and characterization of L-lysine decarboxylase from Huperzia serrata and its role in the metabolic pathway of lycopodium alkaloid. Phytochemistry 2017, doi:10.1016/j. phytochem.2016.12.022.

12. Sun J, Morita H, Chen G, Noguchi H, Abe I. Molecular cloning and characterization of copper amine oxidase from Huperzia serrata. Bioorg Med Chem Lett. 2012;22(18):5784-90.

13. Wanibuchi K, Zhang P, Abe T, Morita H, Kohno T, Chen G, Noguchi H, Abe I. An acridone-producing novel multifunctional type III polyketide synthase from Huperzia serrata. FEBS J. 2007:274(4):1073-82.

14. Morita H, Kondo S, Kato R, Wanibuchi K, Noguchi H, Sugio S, Abe I, Kohno T. Crystallization and preliminary crystallographic analysis of an acridoneproducing novel multifunctional type III polyketide synthase from Huperzia serrata. Acta Crystallogr F. 2007;63(7):576-8.

15. Wang J, Wang X, Liu X, Li J, Shi X, Song Y, Zeng K, Zhang L, Tu P, Shi S. Synthesis of unnatural 2-substituted quinolones and 1, 3-diketones by a member of type III polyketide synthases from Huperzia serrata. Org Lett. 2016;18(15):3550-3.

16. Hemscheidt T, Spenser ID. Biosynthesis of lycopodine: Incorporation of acetate via an intermediate with C2v symmetry. J Am Chem Soc. 1993; 115(7):3020-1.

17. Ma X, Gang DR. The lycopodium alkaloids. Nat Prod Rep. 2004;21(6):752-572.

18. Grabherr MG, Haas BJ, Yassour M, Levin JZ, Thompson DA, Amit I, Adiconis X, Fan L, Raychowdhury R, Zeng Q, Chen Z. Full-length transcriptome assembly from RNA-Seq data without a reference genome. Nat Biotechnol. 2011;29(7):644-52.

19. Langmead B, Salzberg SL. Fast gapped-read alignment with Bowtie 2 . Nat Methods. 2012;9(4):357-9.

20. Katiyar A, Smita S, Lenka SK, Rajwanshi R, Chinnusamy V, Bansal KC. Genome-wide classification and expression analysis of MYB transcription factor families in rice and Arabidopsis. BMC Genomics. 2012;13(1):1.

21. Hartmann U, Sagasser M, Mehrtens F, Stracke R, Weisshaar B. Differential combinatorial interactions of cis-acting elements recognized by R2R3-MYB,
BZIP, and BHLH factors control light-responsive and tissue-specific activation of phenylpropanoid biosynthesis genes. Plant Mol Biol. 2005;57(2):155-71.

22. Rueffer M, Zenk MH. Canadine synthase from Thalictrum tuberosum cell cultures catalyses the formation of the methylenedioxy bridge in berberine synthesis. Phytochemistry. 1994;36(5):1219-23.

23. Yamamoto H, Katano N, Ooi A, Inoue K. Secologanin synthase which catalyzes the oxidative cleavage of loganin into secologanin is a cytochrome P450. Phytochemistry. 2000;53(1):7-12.

24. Ma X, Tan C, Zhu D, Gang DR. Is there a better source of huperzine A than Huperzia serrata? Huperzine A content of Huperziaceae species in China. J Agric food Chem. 2005;53(5):1393-8.

25 Li B, Dewey CN. RSEM: accurate transcript quantification from RNA-Seq data with or without a reference genome. BMC Bioinf. 2011;12(1):1.

\section{Submit your next manuscript to BioMed Central and we will help you at every step:}

- We accept pre-submission inquiries

- Our selector tool helps you to find the most relevant journal

- We provide round the clock customer support

- Convenient online submission

- Thorough peer review

- Inclusion in PubMed and all major indexing services

- Maximum visibility for your research

Submit your manuscript at www.biomedcentral.com/submit
) Biomed Central 